\title{
Attentional load modulates mislocalization of moving stimuli, but does not eliminate the error
}

\author{
DIRK KERZEL \\ University of Geneva, Geneva, Switzerland
}

\begin{abstract}
Localization of the onset and offset of a moving target is subject to a number of errors that have to be attributed to events following or preceding the target event. Apparently, observers are unable to ignore the spatiotemporal context surrounding the target event. In two experiments, observers' attention was directed toward a single position along a trajectory, two positions along a single trajectory, or two positions along two different trajectories. In the latter condition, attention to details of a single trajectory was reduced. At the same time, motion type was manipulated by varying the temporal interval between successive target presentations. The localization error was not affected by attentional load; however, effects of motion type were eliminated when two trajectories had to be attended to. It may be sufficient to notice that the target has moved for localization errors to occur, while specifics of the trajectory are ignored.
\end{abstract}

When observers are asked to localize the first or final position of a moving target, judgments are systematically displaced. When observers are asked to point to the initial position of a moving target, judgments are displaced opposite to the direction of motion (onset repulsion effect; see, e.g., Hubbard \& Motes, 2002; Thornton, 2002). In contrast, judgments of the initial position are displaced in the direction of motion when relative judgments (e.g., left or right of a probe stimulus) are used (Fröhlich effect; Kirschfeld \& Kammer, 1999; Whitney \& Cavanagh, 2002). The onset repulsion effect has been attributed to error avoidance strategies during pointing (Kerzel, 2002a; Thornton, 2002), whereas the Fröhlich effect has been attributed to attentional delays (Kirschfeld \& Kammer, 1999; Müsseler \& Aschersleben, 1998). When observers are asked to judge the final position of a moving target, judgments are displaced in the direction of motion (representational momentum; Freyd \& Finke, 1984; Hubbard, 1995). Representational momentum has been attributed to internalized principles of physical momentum (Freyd \& Finke, 1984), implicit knowledge of physical principles (Hubbard, 1996), belief in naive impetus (Kozhevnikov \& Hegarty, 2001), eye movements (Kerzel, 2000), and attention (Kerzel, 2003a).

Important to the present study is the fact that representational momentum is stronger with apparent (or implied) motion than with smooth motion (Kerzel, 2003b). When the target position is updated at a high rate, the spatiotemporal separation of successive target presenta-

The author was supported by the Deutsche Forschungsgemeinschaft (DFG KE 825/3-1 and 825/4-1,2). I thank Lars Pracejus and Nina Barthels for helping to collect the data. Correspondence concerning this article should be addressed to D. Kerzel, Université de Genève, Uni Mail FaPSE, 40 bd du Pont D'Arve, CH-1205, Geneva, Switzerland (e-mail: dirk.kerzel@pse.unige.ch). tions is too small to be resolved by the visual system. In this case, we see smooth motion of an apparently continuously present target. In contrast, when successive target presentations are separated by large spatiotemporal distances, the resulting impression of motion is weak and is referred to as apparent or implied motion. Even though the impression of motion is stronger with smooth motion than with implied motion, the shift of the final target position in the direction of motion is larger with implied motion. In contrast to offset localization, the effects of motion type on onset localization have not been examined in great detail. Thornton (2002) reported that observers mislocalized the first position with smooth motion, but not when only the first and final positions of the target were shown. However, motion type was not varied systematically.

In general, mislocalization of the initial (or final) target position is due to motion after (or before) the relevant target position. Without a motion trajectory, there would be no displacement in or opposite the direction of motion. What is most stunning about these errors of human performance is that they result from an apparent inability to ignore events that occur before or after the response-relevant event. For instance, representational momentum occurs even though observers know exactly when the relevant position will be presented and there is a large spatiotemporal gap between the relevant event and the context (Freyd \& Finke, 1984). In this situation, observers could, in principle, ignore the preceding events on purely temporal grounds. However, they are unable to do so. The purpose of the present study was to investigate whether the bias introduced by the motion trajectory can be eliminated by dividing attention between two trajectories.

Observers were instructed to memorize a single position, two positions along the same motion trajectory, or two positions along different trajectories. When the ini- 
tial and the final positions of a trajectory have to be memorized, attention has to be spread across the complete trajectory. If only a single position has to be memorized, observers could, in principle, ignore the remaining positions. If two positions along different trajectories have to be memorized, observers' attention is divided between two targets, and processing of a single trajectory will be less efficient, so that parts of the trajectories will be ignored. Thus, variation of the memory set may shed light on whether processing of a trajectory is mandatory and occurs regardless of what the task is or whether a trajectory can be ignored if task requirements favor such a strategy.

To further evaluate whether observers process details of a motion trajectory, motion type was manipulated. Effects of motion type would indicate that the observers had processed the trajectory, whereas their absence would indicate that the trajectory had not been fully processed. Either smooth motion or various types of apparent and implied motion were presented. To this end, the stimulus onset asynchrony (SOA) between successive target presentations was varied. With the smallest SOA $(27 \mathrm{msec})$, the resulting motion looked smooth; with medium SOAs (80-160 msec), apparent motion resulted; and motion was merely implied with SOAs larger than $320 \mathrm{msec}$. Previous studies have shown that representational momentum is larger with apparent or implied motion than with smooth motion (Kerzel, 2003b). Similarly, onset repulsion has been found to be absent with implied motion (Thornton, 2002), indicating that relative to smooth motion, implied motion produced forward displacement of the judged onset.

So far, the effects of attention on memory displacement have been looked at in three studies. First, Hayes and Freyd (2002) investigated memory for the final position of a target undergoing implied motion. When observers had to simultaneously attend to the size of another object, the forward shift of the final position of a moving target increased. Similarly, when observers had to perform a verbal task while memorizing the final target position, the forward shift increased. Thus, dividing attention between two objects or two tasks increased representational momentum. Second, Kerzel (2003a) found that a distracting object presented during the retention interval eliminated representational momentum. These two findings appear contradictory but may be reconciled by considering the different ways to manipulate attention: Visual distractors in Kerzel (2003a) captured visuospatial attention because of their sudden onset but were task irrelevant otherwise, whereas a second verbal or visual task in Hayes and Freyd induced a split focus of attention. Thus, attention was taxed differently in these studies, and it may be that this had different effects on the processes underlying representational momentum. Third, Hubbard and Motes (2002) asked observers to indicate either the first or the final target position and manipulated speed and direction of the smoothly moving target. They found that the effects of velocity and direction were less pronounced when the observers had to at- tend to both the onset and the offset position, as compared with a condition in which the observers attended to only one position. However, they did not statistically confirm these results.

\section{EXPERIMENT 1}

In Experiment 1, one group of observers was asked to memorize both the onset and the offset positions, whereas the other group was asked to memorize only one position at a time. To ensure that the observers attended to both the onset and the offset positions, the former group was not told which target position was to be judged until the target had vanished. The other group was told beforehand whether they were to judge the onset or the offset position. The observers judged the onset or offset position by adjusting a mouse cursor.

\section{Method}

Participants. A total of 21 students at the Justus-Liebig-University of Giessen were paid for their participation. The participants reported normal or corrected-to-normal vision and were naive as to the purpose of the experiment.

Stimuli and Apparatus. The stimuli were presented on a 17-in. (diagonal) display with a resolution of $1,152(\mathrm{H}) \times 864(\mathrm{~V})$ pixels and a refresh rate of $75 \mathrm{~Hz}$. One pixel measured $2.1 \mathrm{~min}$ of arc. Target and background luminance was 0.5 and $43.3 \mathrm{~cd} / \mathrm{m}^{2}$, respectively. The position of one eye was monitored with a head-mounted, video-based eye tracker (EyeLink II, SR Research, Osgoode, ON, Canada). The observers' head position was stabilized with a chinrest. Black disks with diameters of 42 and 12 arcmin were used as target stimulus and fixation mark, respectively. The target moved on a circular orbit with a radius of $3^{\circ}$ of visual angle at a velocity of $3.1 \% \mathrm{sec}$. SOAs were approximately $27,80,160,320$, and $640 \mathrm{msec}$, so that the target position was changed at a frequency of 37.55 , $12.5,6.25,3.13$, and $1.56 \mathrm{~Hz}$. Presentation time and blank intervals were half the SOA. The trajectory length measured $4^{\circ}$, which is equivalent to $76^{\circ}$ of rotation. A straight line between the start- and the endpoints of the trajectory measured $3.7^{\circ}$, and the maximal deviation orthogonal to this line was $0.6^{\circ}$, so that the curvature of the trajectory was rather small. Presentation time varied as a function of SOA (see Figure 1).

Procedure and Design. The observers sat about $45 \mathrm{~cm}$ in front of the computer screen. Eye fixation was monitored and had to be maintained within $1^{\circ}$ of the central fixation mark. The observers were instructed to maintain fixation during target presentation. Immediately after target offset, the fixation mark changed its color, and $280 \mathrm{msec}$ later, a white, crosshair mouse cursor $(23 \times 23 \mathrm{arcmin})$ appeared on the central fixation mark. The observers were instructed to click on the initial position of the moving target when the fixation dot turned green and to click on the final position when it turned red. For 10 observers, the task (onset vs. offset localization) varied unpredictably from trial to trial. For another 11 observers, the task was blocked, and the order of blocks was approximately counterbalanced across observers. The five different motion types were shown randomly interleaved. Starting position and direction of rotation were random. The observers worked through two blocks of 120 trials each that were composed of 24 repetitions of the 10 possible combinations of SOA and task. The predictability of the task (random vs. blocked) was varied between subjects.

\section{Results}

Deviations of the judged from the true final position were determined along the tangent to the orbit (similar 


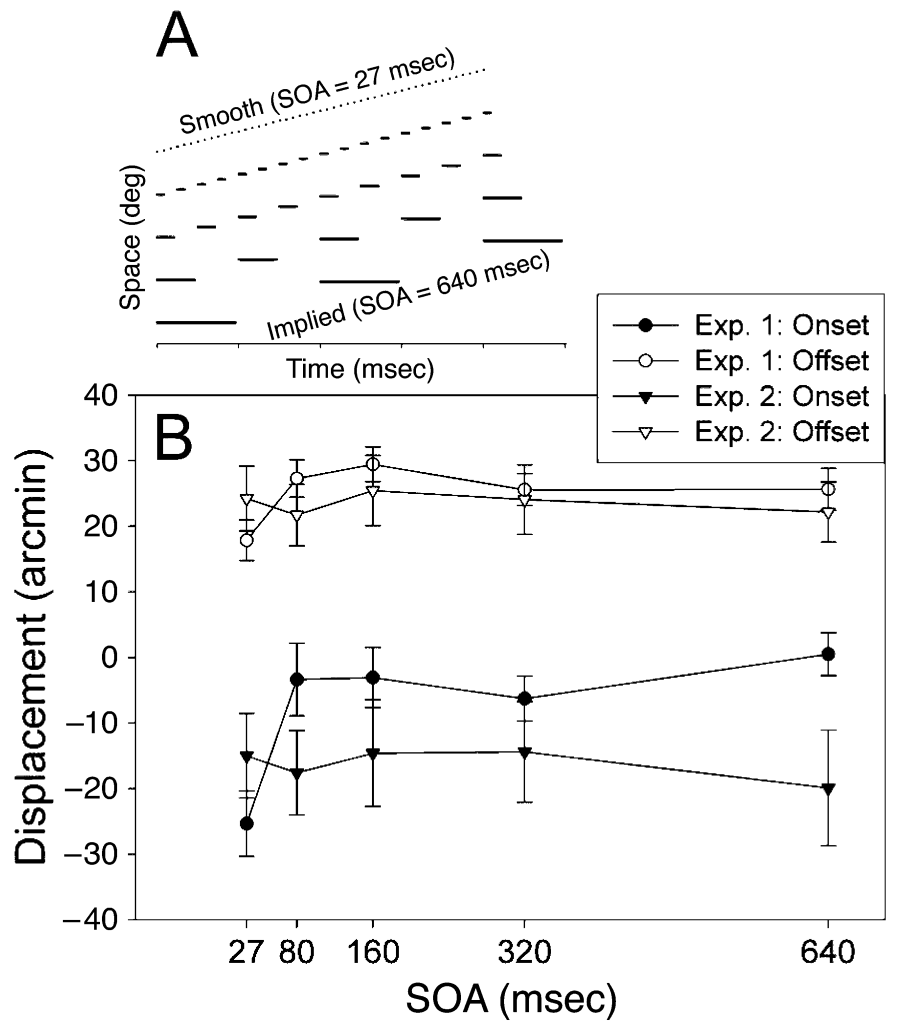

Figure 1. (A) A space-time plot of the different motion types. With implied motion, successive stimulus presentations are separated by large spatiotemporal gaps. Perceptually, the stimulus appears at successive positions, and the impression of motion is weak. With smooth motion, the spatiotemporal gaps are small, and the target appears to be continuously present. Implied and smooth motions mark the extremes of a continuum that varies along the stimulus onset asynchrony (SOA). (B) Effects of SOA on displacement in Experiments 1 and 2. Positive and negative displacements indicate that the observers mislocalized the target in and opposite the direction of motion, respectively. The observers were asked to judge either the first position of the moving target (onset) or its final position (offset).

methods were used by Hubbard, 1996, and Kerzel, 2003b). Positive and negative displacement values indicate that the judged position deviated in and opposite to, respectively, the direction of motion from the true position. Deviations orthogonal to the tangent were not considered, since these errors reflect biases to localize toward the fovea (Kerzel, 2002b; Sheth \& Shimojo, 2001) and effects of the initial cursor position (van der Heijden, van der Geest, de Leeuw, Krikke, \& Müsseler, 1999).

Due to eye movements or blinks, $4.6 \%$ of the trials were excluded. A mixed-factors analysis of variance (ANOVA; task predictability $\times$ task $\times$ SOA) on displacement did not show any effects of task predictability ( $p$ s $>.28$ ) but did show a significant increase with increases in SOA $\left[F(4,76)=17.30, M S_{\mathrm{e}}=123.56, p<\right.$ $.0001]$. Displacement was smaller with onset than with offset localization ( -8 vs. 25 arcmin; $F(1,19)=40.92$, $\left.M S_{\mathrm{e}}=1,366.90, p<.0001\right]$. The effect of SOA was modulated by task $\left[F(4,76)=3.69, M S_{\mathrm{e}}=127.97, p<\right.$ .01]. Separate one-way ANOVAs confirmed that the effect of SOA was significant for both onset $[F(4,84)=$ $\left.11.99, M S_{\mathrm{e}}=184.54, p<.0001\right]$ and offset $[F(4,84)=$ 6.31, $\left.M S_{\mathrm{e}}=63.43, p<.0001\right]$ localization. Contrasts confirmed that the effects of SOA were due mainly to a significant difference between the smallest SOA and the remaining SOAs (all $p \mathrm{~s}<.01$ ). See Table 1 for $t$ tests comparing the means of each combination of task and SOA.

\section{Discussion}

There were strong effects of motion type that were not modulated by whether the observers attended to both the onset and the offset positions or to only one position. Smooth motion produced more displacement opposite to motion when the onset was judged and less displacement in the direction of motion when the offset was judged. It 
Table 1

Mean Displacement in Minutes of Arc as a Function of Task (Onset vs. Offset Localization) and Stimulus Onset Asynchrony (SOA) in Experiments 1 and 2

\begin{tabular}{cccccc}
\hline & \multicolumn{2}{c}{ Experiment 1} & & \multicolumn{2}{c}{ Experiment 2} \\
\cline { 2 - 3 } \cline { 5 - 6 } SOA $(\mathrm{msec})$ & Onset & Offset & & Onset & Offset \\
\hline 27 & $-25^{* * *}$ & $18^{* * *}$ & & $-15^{*}$ & $24^{* *}$ \\
80 & -3 & $27^{* * *}$ & & $-18^{*}$ & $22^{* *}$ \\
160 & -3 & $29^{* * *}$ & & $-15 \dagger$ & $25^{* *}$ \\
320 & $-6 \dagger$ & $26^{* * *}$ & & $-14 \dagger$ & $24 * *$ \\
640 & 0 & $26^{* * *}$ & & $-20^{*}$ & $22^{* *}$ \\
$M$ & $-8^{*}$ & $25^{* * *}$ & & $-16^{*}$ & $24 * *$ \\
\hline
\end{tabular}

Note-Each mean was compared with zero by $t$ test. The level of significance is indicated by different symbols. Probabilities smaller than or equal to .10 are indicated by $\dagger$, those smaller than .05 by $*$, those smaller than .001 by ${ }^{* *}$, and those smaller than .0001 by $* * *$.

is surprising that only smooth motion produced onset repulsion, whereas the onset of apparent or implied motion stimuli was correctly judged. That is, even a slight deviation from smooth motion (i.e., with an SOA of $80 \mathrm{msec}$ ) eliminated onset repulsion. Similarly, the judged offset of smooth motion was displaced opposite to motion, relative to apparent or implied motion. However, the bias to mislocalize the offset in the direction of motion was significant across motion types.

In sum, the present results suggest that localization of objects in smooth motion differs from that for other motion types. Judgments of the onset and the offset were shifted backward relative to apparent or implied motion, so that onset repulsion resulted when the onset was localized, whereas a smaller forward shift resulted when the offset was localized. However, localization performance was unaffected by attentional load. Regardless of whether the observers had to attend to both onset and offset or only one position, smooth motion produced less forward and more backward displacement than did apparent or implied motion. Similarly, the overall size of the displacement was unaffected by memory load. Thus, one may conclude that the observers attended to the full trajectory even if only one position had to be judged.

\section{EXPERIMENT 2}

In Experiment 2, the observers were asked to judge the onset or offset of one of two moving objects. The two targets moved in synchrony, but the starting position and direction of motion were randomized. After target offset, a triangle pointed to the target that was to be localized. To clearly separate the two targets, linear motion, instead of circular motion, was used. One target moved in the upper visual field; the other moved in the lower. A previous study had shown that attending to another expanding or shrinking object increases representational momentum for linear motion (Hayes \& Freyd, 2002). If divided attention increases biases in localization, both onset repulsion and representational momentum would be expected to increase in size in Experiment 2. If, on the other hand, divided attention helps observers to ignore aspects of a trajectory, onset repulsion and representational momentum would be reduced. Effects of motion type would serve as an index of whether the observers fully processed the trajectory. If the trajectory was fully processed, the effects of motion type should be the same as those in Experiment 1.

\section{Method}

Participants. Twelve students fulfilling the same criteria as those in Experiment 1 participated.

Stimuli, Apparatus, Design, and Procedure. The stimuli, apparatus, design, and procedure were the same as those in Experiment 1 , with the following exceptions. Two targets were shown: one above the fixation point and one below. Their trajectories were linear and horizontal, but the remaining characteristics of the motion were the same as those in Experiment 1. The vertical offset of the targets was 106 arcmin, and the horizontal offset was random within 120 arcmin of the fixation point. In half of the trials, the two targets moved in the same direction; in the other half, they moved in opposite directions. Immediately after target offset, an upward- or downward-pointing triangle was shown at fixation, and the observers were asked to localize the target located on the side the triangle was pointing to. The cursor appeared $280 \mathrm{msec}$ after target offset at fixation. The localization task (onset vs. offset) was blocked, and the observers worked through 240 trials per task. The tasks were run on different days.

\section{Results}

Displacement was defined as the difference between true and judged onset along the linear direction of motion. Due to eye movements or blinks, $2.6 \%$ of the trials had to be excluded. A two-way ANOVA (task $\times$ SOA) showed that displacement was smaller with onset than with offset localization [-16 vs. 24 arcmin; $F(1,11)=12.77, M S_{\mathrm{e}}=$ $3,720.87, p<.005]$. No other effects were significant $(p s>.14)$. Separate one-way ANOVAs confirmed that the effect of SOA was absent with onset $(p>.44)$ and offset $(p>.37)$ localization. A further three-way betweensubjects ANOVA (experiment $\times$ task $\times$ SOA) evaluated differences between Experiments 1 and 2. There were significant effects of task $\left[F(1,30)=45.99, M S_{\mathrm{e}}=2,230.03\right.$, $p<.0001]$ and SOA $\left[F(4,120)=13.18, M S_{\mathrm{e}}=95.5, p<\right.$ $.0001]$, a significant interaction between task and SOA $\left[F(4,120)=2.54, M S_{\mathrm{e}}=99.5, p<.05\right]$, and importantly, a significant interaction between SOA and experiment $\left[F(8,120)=5.39, M S_{\mathrm{e}}=95.5, p<.0001\right]$.

\section{Discussion}

The effect of motion type was eliminated when two objects had to be attended to. In contrast to Experiment 1, smooth motion did not produce memory displacement that differed from that with apparent or implied motion. This suggests that details of the trajectory (i.e., motion type) were not processed to the same degree as in Experiment 1 . However, onset repulsion and representational momentum persisted, suggesting that the trajectory could not be ignored. One may argue that the different effects of SOA in Experiments 1 and 2 were due to differences between linear (Experiment 1) and rotational (Experi- 
ment 2) motion. However, this argument is not convincing. First, the curvature of the trajectory in Experiment 1 was rather small, so that the differences between linear and circular trajectory were negligible. Second, across studies, the effects of motion type have mirrored those in Experiment 1 and have appeared to be independent of trajectory shape. Onset repulsion was larger with smooth than with implied motion along linear trajectories (Thornton, 2002). Representational momentum was observed with implied motion along circular (e.g., Freyd \& Johnson, 1987) and linear (e.g., Hayes \& Freyd, 2002) trajectories and was clearly absent with smooth motion along circular (Kerzel, 2003b) and linear (Kerzel, 2000) trajectories. Third, an explicit comparison did not yield significant differences between linear and circular motion for onset localization (Kerzel, 2002a).

The comparison of Experiments 1 and 2 showed that attending to a second object did not affect the magnitude of representational momentum or onset repulsion (i.e., there was no interaction of experiment and task). In contrast, Hayes and Freyd (2002) have reported that attention to a second object increased representational momentum. However, Hayes and Freyd used probe judgments, which may produce different results than does mouse pointing. Furthermore, onset repulsion was not significantly different in Experiment 2 from that in Experiment 1 even though the effect was numerically larger (see Table 1). It may be that the relatively large variability between participants (see Figure 1) overshadowed this difference in the between-subjects design. However, it should be noted that onset repulsion was reliable across most SOAs in Experiment 2, whereas only smooth motion produced onset repulsion in Experiment 1.

\section{GENERAL DISCUSSION}

In two experiments, the effects of memory load and motion type on the localization of the first and final positions of a moving target were investigated. Memory load was manipulated by asking observers to memorize one or two positions along a single trajectory or two positions along two trajectories. The rationale was that memorizing more than one position along the same trajectory would increase attention to the trajectory, whereas attention to only one position would decrease attention to the trajectory. Similarly, if observers had to memorize two positions along two different trajectories, attention to a single trajectory would be reduced. Two indices for attention to the target's trajectory were used. First, the deviation of the judged from the actual position of the target was determined. If the observers were able to ignore the trajectory, the typical biases in and opposite to motion should be reduced. Second, effects of motion type on memory displacement were determined. The results showed that the observers could not ignore the trajectory, because there was significant displacement in and opposite the direction of motion regardless of atten- tional load. However, the results also showed that details of the trajectory were not processed when two trajectories were attended to. When attention was divided between two trajectories, effects of motion type disappeared. Thus, the conclusion is that for displacement of the initial or final position to occur, the observer only needs to notice that the target moved. It does not matter very much how it moved. Even if very little attention is devoted to the trajectory, there will be displacement of the initial and final target positions. However, effects of motion type depend on the observer's attention being assigned to a single trajectory.

Furthermore, the study showed that type of motion strongly influenced object localization — at least when only a single trajectory was attended to. The most pronounced difference was that observed between the smooth and the remaining motion types (apparent and implied). Onset and offset judgments with smooth motion showed displacement opposite to motion, relative to apparent and implied motion. Thus, smooth motion produced onset repulsion and less representational momentum with offset localization. There are at least two interpretations of this effect. First, it may be that the observers automatically predicted the next step of the motion trajectory. With implied or apparent motion stimuli, the next step would be larger than that with smooth motion. In fact, the next step with smooth motion would be very close to zero. If judgments were biased toward the predicted next step of the sequence or if the observers confounded the next step with the final position, larger forward displacement would occur with apparent and implied motion than with smooth motion (see also Kerzel, 2003b). However, there was no increase in representational momentum beyond the $80-\mathrm{msec}$ SOA, suggesting that there is an upper limit to this confusion. Second, it may be that the observers tried to avoid obvious errors during pointing movements (see also Thornton, 2002). With smooth motion, the onset and offset positions were less clear than with apparent motion, because they were presented for only very short periods of time (i.e., $13 \mathrm{msec}$ vs. longer than $40 \mathrm{msec}$ ). Thus, the observers may have been less certain about when and where the object appeared or vanished. As a result, they may have compensated for the uncertainty by correcting for the most obvious error: being too late. When they were asked to point to the onset, this resulted in onset repulsion; when they were asked to point to the offset, this resulted in reduced representational momentum.

In sum, representational momentum and onset repulsion were unaffected by whether one position, a complete trajectory, or two trajectories had to be attended to. However, effects of motion type were clearly present when attentional load was low (with one position or one trajectory) but absent when it was high (with two trajectories). Thus, onset repulsion and representational momentum may occur even if details of the trajectory are not fully processed. 


\section{REFERENCES}

Freyd, J. J., \& FinKe, R. A. (1984). Representational momentum. Journal of Experimental Psychology, 10, 126-132.

Freyd, J. J., \& Johnson, J. Q. (1987). Probing the time course of representational momentum. Journal of Experimental Psychology: Learning, Memory, \& Cognition, 13, 259-268.

HAYES, A. E., \& FreYD, J. J. (2002). Representational momentum when attention is divided. Visual Cognition, 9, 8-27.

HuBbard, T. L. (1995). Environmental invariants in the representation of motion: Implied dynamics and representational momentum, gravity, friction, and centripetal force. Psychonomic Bulletin \& Review, 2, 322-338

HubBard, T. L. (1996). Representational momentum, centripetal force, and curvilinear impetus. Journal of Experimental Psychology: Learning, Memory, \& Cognition, 22, 1049-1060.

Hubbard, T. L., \& Motes, M. A. (2002). Does representational momentum reflect a distortion of the length or the endpoint of a trajectory? Cognition, 82, B89-B99.

KerZEL, D. (2000). Eye movements and visible persistence explain the mislocalization of the final position of a moving target. Vision Research, 40, 3703-3715.

KERZEL, D. (2002a). Different localization of motion onset with pointing and relative judgements. Experimental Brain Research, 145, 340350 .

Kerzel, D. (2002b). Memory for the position of stationary objects: Disentangling foveal bias and memory averaging. Vision Research, 42, $159-167$.
Kerzel, D. (2003a). Attention maintains mental extrapolation of target position: Irrelevant distractors eliminate forward displacement after implied motion. Cognition, 88, 109-131.

KerZEL, D. (2003b). Mental extrapolation of target position is strongest with weak motion signals and motor responses. Vision Research, $\mathbf{4 3}_{2}$ 2623-2635.

Kirschfeld, K., \& Kammer, T. (1999). The Fröhlich effect: A consequence of the interaction of visual focal attention and metacontrast. Vision Research, 39, 3702-3709.

KoZHeVNIKOV, M., \& HegarTy, M. (2001). Impetus beliefs as default heuristics: Dissociation between explicit and implicit knowledge about motion. Psychonomic Bulletin \& Review, 8, 439-453.

MüsSELER, J., \& ASCHERSLEBEN, G. (1998). Localizing the first position of a moving stimulus: The Fröhlich effect and an attention-shifting explanation. Perception \& Psychophysics, 60, 683-695.

Sheth, B. R., \& Shimojo, S. (2001). Compression of space in visual memory. Vision Research, 41, 329-341.

Thornton, I. M. (2002). The onset repulsion effect. Spatial Vision, 15, 219-244.

van der Heijden, A. H., van der Geest, J. N., de Leeuw, F., Krikke, K., \& MÜsSELER, J. (1999). Sources of position-perception error for small isolated targets. Psychological Research, 62, 20-35.

Whitney, D., \& CAVAnaGH, P. (2002). Surrounding motion affects the perceived locations of moving stimuli. Visual Cognition, 9, 139-152.

(Manuscript received July 28, 2003;

revision accepted for publication November 24, 2003.) 